\title{
Biochanin A alleviates gingival inflammation and alveolar bone loss in rats with experimental periodontitis
}

\author{
SHENGDAN ZHANG ${ }^{1,2^{*}}$, YULONG NIU ${ }^{3 *}$, ZHUO YANG $^{4}$, YUWEI ZHANG ${ }^{1,2}$, QIANG GUO $^{1}$, \\ YI YANG ${ }^{3}$, XUEDONG ZHOU ${ }^{1,5}$, YI DING ${ }^{2}$ and CHENGCHENG LIU ${ }^{2}$ \\ ${ }^{1}$ State Key Laboratory of Oral Diseases; ${ }^{2}$ Department of Periodontics, West China Hospital of Stomatology; \\ ${ }^{3}$ Key Laboratory of Bio-Resources and Eco-Environment of Ministry of Education, College of Life Sciences, \\ Sichuan University, Chengdu, Sichuan $610041 ;{ }^{4}$ General Stomatology Clinic, Stomatological Hospital of Chongqing \\ Medical University, Chongqing 401147; ${ }^{5}$ Department of Operative Dentistry and Endodontics, \\ West China Hospital of Stomatology, Sichuan University, Chengdu, Sichuan 610041, P.R. China
}

Received December 14, 2019; Accepted May 20, 2020

DOI: $10.3892 /$ etm.2020.9381

\begin{abstract}
Biochanin A (BA) is an organic compound produced by Trifolium pretense and Arachis hypogaea with anti-inflammatory and antioxidative effects. The aim of the current study was to evaluate the effects of BA on gingival inflammation and alveolar bone destruction in rats with experimental periodontitis. Experimental rats $(n=25)$ were distributed equally into five groups: i) Healthy control (control) group; ii) experimental periodontitis (ligation) group; and iii) and ligation plus low, medium and high dose of BA $(12.5,25$ and $50 \mathrm{mg} / \mathrm{kg} /$ day, respectively) groups. A nylon ligature was inserted around rats' maxillary molars for 14 days to trigger the experimental periodontitis. BA was intravenous injected once daily for 4 weeks. After that, interleukin-1 $\beta$ (IL-1 $\beta$ ), tumor necrosis factor- $\alpha$ (TNF- $\alpha$ ), reactive oxygen species (ROS) and osteocalcin (OCN) levels were determined in gingival and/or serum samples using ELISA or reverse transcription-quantitative PCR. Alveolar bone volume was assessed via hematoxylin and eosin staining and micro-computed tomography. Osteoclasts were identified by tartrate-resistant acid phosphatase staining, and the level of the nuclear factor erythroid-2 related factor 2 (Nrf2) was also detected by immunohistochemical staining. BA treatment groups showed alleviated alveolar bone resorption compared with the ligation group. Moreover, BA
\end{abstract}

Correspondence to: Professor Yi Ding or Professor Chengcheng Liu, Department of Periodontics, West China Hospital of Stomatology, Sichuan University, 14, Section 3, Renmin South Road, Chengdu, Sichuan 610041, P.R. China

E-mail: yiding2000@126.com

E-mail: liuchengcheng519@163.com

${ }^{*}$ Contributed equally

Key words: biochanin A, periodontitis, inflammation, reactive oxygen species, bone loss treatment significantly inhibited IL-1 $\beta$, TNF- $\alpha$, ROS levels, and reduced leukocyte acid phosphatase-positive cells, as well as increased OCN and Nrf2 levels compared with the ligation group. BA had beneficial effects on experimental periodontitis of rats. BA treatment inhibited inflammation, regulated unbalanced oxidative stress response and ameliorated the alveolar bone loss.

\section{Introduction}

Biochanin A (BA; chemical formula, $\mathrm{C}_{16} \mathrm{H}_{12} \mathrm{O}_{5}$ ), is a phytochemical and a flavonoid present in natural produce such as Trifolium pratense and Arachis hypogaea (1). BA exhibits various protective effects including anti-inflammatory, glucose and lipid metabolism modulatory activity, antitumor capacity, and neuroprotective activity (2-4). BA can inhibit the lipopolysaccharide (LPS)-induced activation of microglia (5). The anti-inflammatory activity of BA has been demonstrated in a variety of cells such as endothelial cells, various cancer cells and macrophages $(6,7)$. Studies in vitro have shown that the proliferation of preosteoclast cells was markedly inhibited in a dose-dependent manner in the presence of BA. Furthermore, BA promoted the differentiation of primary osteoblasts into osteoblasts, and significantly enhanced the expression and activity of alkaline phosphatase (ALP) (4). Mature osteoblast migration and the number of mineralized nodules, consisting of phosphorus and calcium are significantly increased by BA (4). Furthermore, it has been reported that BA decreased the $\mathrm{H}_{2} \mathrm{O}_{2}$-induced production of TNF- $\alpha$, interleukin (IL)-6 and nitric oxide (NO) in osteoblasts (8). The anti-inflammatory effects of BA are mediated by inhibiting the inflammatory mediators, includeing IL-1 $\beta$, IL-6, TNF- $\alpha$, cyclooxygenase-2 (COX-2), matrix metalloprotein-9 (MMP-9) and NO in primary rat chondrocytes (9). BA decreases the expression of proinflammatory cytokines by inhibiting $\mathrm{I} \kappa \mathrm{B}$ kinase activity, resulting in NF- $\mathrm{KB}$-driven inhibition of gene transcription $(8,10,11)$. Furthermore, BA can exert the antioxidative activity by scavenging reactive oxygen species (ROS) and enhancing the glutathione peroxidase and superoxide dismutase activity (12). A previous study conducted using 
animal models with acute and chronic inflammation demonstrated a marked anti-inflammatory and antioxidative activity of BA against organ injury (13). BA-mediated inhibition of bone loss in postmenopausal women has also been reported in a previous study (14). These findings suggested that BA treatment can potentially regulate bone cell proliferation, apoptosis and migration, leading to reduced bone loss and bone turnover.

Periodontitis is a prevalent chronic disease worldwide. The 1990-2016 Global Burden of Disease Study indicated that periodontitis and dental caries in permanent teeth were the leading and the 11th most prevalent diseases worldwide in 2016. Periodontal diseases had a high prevalence among all human diseases (751 million; 95\% uncertainty interval, 534-874 million) (15). Periodontitis is characterized by the breakdown of connective tissues and alveolar bone resorption eventually leading to mobility and loss of teeth (16). An inappropriate host immune inflammatory response and high levels of ROS are considered major factors associated with the destruction of periodontal tissues (17). Elevated levels of proinflammatory cytokines, such as IL- $1 \beta$ and TNF- $\alpha$, trigger soft and hard tissue breakdown in periodontitis (18). Moreover, previous studies have suggested a dual role of ROS in periodontitis $(19,20)$. At a cellular level, ROS are required for physiological processes and contribute to the oxidative killing of periodontal pathogens. However, excessive production of ROS may trigger an oxidative stress response resulting in periodontal destruction. In addition, oxidative stress response can reinforce immune response through redox-sensitive gene transcription factors such as $\mathrm{NF}-\kappa \mathrm{B}$, leading to periodontal tissue breakdown (21). Therefore, anti-inflammatory and anti-oxidative responses are essential for the treatment of periodontitis.

The biological effects of BA and the mechanisms involved in the pathogenesis of periodontitis suggest that BA may serve as an adjunctive treatment option for periodontitis via the anti-inflammatory and anti-oxidative effects. Therefore, the aim of the present study was to evaluate the influence of BA on gingival inflammation and tissue destruction in rat periodontitis. In addition, the effects of BA supplementation on the expression levels of periodontal inflammatory markers and oxidative stress were explored.

\section{Materials and methods}

Ethics approval. The present study was approved by Ethics Committee of the State Key Laboratory of Oral Diseases, West China Hospital of Stomatology, Sichuan University (approval no. WCHSIRB-D-2018-148).

Animals. The animal experimental study was conducted using 26 male Wistar rats (age, 8 weeks; weight, 280-300 g) purchased from the Chengdu Dashuo Experimental Animal Co., Ltd. (license no. 510109000176387). The rats were housed under 12-h light/dark cycles and standard conditions of humidity $(60 \pm 5 \%)$ and room temperature $\left(24 \pm 1^{\circ} \mathrm{C}\right)$. All animals had free access to food and water. The rats adapted to the environment for 7 days prior to further experiments. One rat was used for isolation of gingival fibroblasts and 25 were used to construct animal models, as described below.
Primary culture of rat gingival fibroblasts. To isolate primary cultures of rat gingival fibroblasts, one Wistar rat was anesthetized with isoflurane (5\% induction, $2 \%$ maintenance; inhaled until euthanized). Gingival tissues were subsequently excised from the buccal area, submersed in sterile PBS with $1 \%$ penicillin/streptomycin (Sigma Aldrich; Merck KGaA), and then sectioned into small fragments of $\sim 1 \mathrm{~mm}^{3}$. The gingival fragments were digested with $2 \mathrm{ml}$ type I collagenase solution in PBS (25 U/ml; Sigma Aldrich; Merck KGaA) at $37^{\circ} \mathrm{C}$ for $1 \mathrm{~h}$, followed with a $15-\mathrm{min}$ incubation at $37^{\circ} \mathrm{C}$ in trypsin-EDTA solution (0.25\%; Thermo Fisher Scientific, Inc.). Subsequently, the fragments were incubated in DMEM with $10 \%$ FBS (both Sigma Aldrich; Merck KGaA) and $1 \%$ penicillin/streptomycin at $37^{\circ} \mathrm{C}(22)$. The cultured cells from gingiva were tested by immunocytochemical analysis. These cells were tested negative for anti-keratin staining whilst staining positive for vimentin, suggesting these cells to be gingival fibroblasts (23). Cells between sixth and ninth passages were used for the assessment of cell cytotoxicity.

Cell cytotoxicity assay. The cytotoxicity of BA (purity, 99.9\%; Sigma Aldrich; Merck KGaA) on rat gingival fibroblasts was evaluated using an MTT assay (Sigma Aldrich; Merck KGaA) according to manufacturer's instructions. Briefly, rat gingival fibroblast cells at a density of $2 \times 10^{4} /$ well were cultured in 96-well plates at $37^{\circ} \mathrm{C}$ for 24 and $48 \mathrm{~h}$ and then treated with 0 , 50, 100 and $150 \mu \mathrm{M}$ BA for $24 \mathrm{~h}$. Subsequently, $20 \mu \mathrm{l}$ MTT was added into each well and incubated for another $4 \mathrm{~h}$ before the supernatant was removed. Finally, $150 \mu \mathrm{l}$ DMSO was added to each well and the absorbance values at a wavelength of $570 \mathrm{~nm}$ were measured using a microplate reader (24).

Study design. A total of 25 Wistar rats were randomly assigned to five study groups: i) Healthy control (control); ii) experimental periodontitis (ligation); iii) ligation $+\mathrm{BA}$ low dose (12.5 mg/kg/day); iv) ligation + BA medium dose ( $25 \mathrm{mg} / \mathrm{kg} /$ day); and v) ligation + BA high dose (50 mg/kg/day). The control group received sham-ligation. For the ligation groups, periodontitis was induced by inserting a nylon ligature around maxillary molars for 14 days as described previously (25). The ligatures were placed sub-gingivally, knotted at the mesial site and checked on a daily basis. Rats in the ligation + BA group were administered with BA (purity, 99.9\%; Sigma Aldrich; Merck KGaA) 2 weeks after establishment of the experimental periodontitis model intraperitoneally at low, medium and high doses, respectively. Control and ligation groups' rats were injected $0.9 \%$ sodium chloride instead of BA. An automatic electronic balance [cat. no. PX4202 OHAUS Instruments (Shanghai) Co., Ltd.] was used to monitor and record rat body weights on a weekly basis. After treatment with BA for 4 weeks, all rats were anesthetized with isoflurane. After deep anesthesia of the rats, blood was drawn by cardiac puncture with non-anticoagulant vacuum tubes and $23 \mathrm{G} 1$ needle. The anesthesia was checked by lack of spontaneous movements, slow breathing rate and lack of response to stimuli. The rats were euthanized immediately at the end of cardiac puncture. The maxillary jaws were harvested and fixed in $10 \%$ neutral-buffered formalin at room temperature $\left(24 \pm 1^{\circ} \mathrm{C}\right)$ for $24 \mathrm{~h}$. The gingival tissues were collected for subsequent analysis. All samples were stored at $-80^{\circ} \mathrm{C}$. 
Determination of inflammatory cytokines, ROS and osteocalcin $(O C N)$. In order to isolate serum, the clotted blood samples were centrifuged (10 min; 2432 x g). Gingival tissues were homogenized with a glass pestle in PBS supplemented with RIPA lysis buffer (Sigma Aldrich; Merck KGaA) and protease inhibitor (Thermo Fisher Scientific, Inc.). Bicinchoninic acid assay kit (Thermo Fisher Scientific, Inc.) was used for protein quantitation. Protein levels of IL-1 $\beta$ and TNF- $\alpha$ in gingiva were measured by ELISA kits (cat. nos. BMS630 and ERA57RB; Invitrogen; Thermo Fisher Scientific, Inc.) according to the manufacturer's instructions. The present study used TRIzol ${ }^{\circledR}$ reagent (Invitrogen; Thermo Fisher Scientific, Inc.) to extract total RNA from the gingival tissues around the maxillary first molars. The PrimeScript Reagent kit with gDNA Eraser (cat. no. RR047A, Takara Bio, Inc.) was used for the reverse transcription of total RNA into cDNA (temperature protocol: $37^{\circ} \mathrm{C}$ for $15 \mathrm{~min}$ followed by $85^{\circ} \mathrm{C}$ for $5 \mathrm{sec}$ ). Quantitative PCR analysis were performed by use of SYBR ${ }^{\circledR}$ Premix Ex Taq $^{\mathrm{TM}}$ II Kit (cat. no. DRR820A, Takara Bio, Inc.) with the CFX96 Touch $^{\text {TM }}$ Real-time PCR system (Bio-Rad Laboratories, Inc.) using the following thermocycling conditions: Initial denaturation at $95^{\circ} \mathrm{C}$ for $30 \mathrm{sec}$, followed by 40 cycles of $95^{\circ} \mathrm{C}$ for $5 \mathrm{sec}$ and $60^{\circ} \mathrm{C}$ for $30 \mathrm{sec}$. IL- $1 \beta$ and TNF- $\alpha$ expression levels in gingival tissues were evaluated using GAPDH internal control and relative quantitative analysis was performed using the $2^{-\Delta \Delta \mathrm{Cq}}$ method (26). Primers for the qPCR are provided in Table SI.

Histological analysis. For histological analysis, the alveolar bone tissue were decalcified using 10\% EDTA at room temperature for 8 days, embedded in paraffin and cut into $4 \mu \mathrm{m}$-thick sections. After heating at $60^{\circ} \mathrm{C}$ for $30 \mathrm{~min}$, xylene was used to deparaffinize the sections $(4-\mu \mathrm{m}$ thick) and gradient ethanol solutions (100, 95, 80 and $70 \%$ ) were used in a descending order to rehydrate the samples, followed by rinsing with deionized water. Sections were stained using hematoxylin and eosin at room temperature for $3 \mathrm{~min}$ and histopathological analysis of six randomly selected fields of view (100x100 $\mu \mathrm{m})$ for each specimen was performed as reported previously (27).

Micro-computed tomography (micro-CT) analysis. A high-resolution micro-CT system ( $\mu$ CT 50; SCANCO Medical AG) was used to scan maxillary jaw samples isolated from the rats, which were harvested and fixed in $10 \%$ neutral-buffered formalin at room temperature for $24 \mathrm{~h}$. Fixed parameters (image size, 2,048x2,048 pixels; voltage, $100 \mathrm{kV}$; electrical current, $0.1 \mathrm{~mA}$; resolution, $10 \mu \mathrm{m}$ ) were used for scanning. The scanned image data and $3 \mathrm{D}$ reconstructions were analyzed by CT-Analyser 1.13 software (SCANCO Medical AG). The vertical loss of bone in the maxillary first molar was assessed by calculating distance $(\mu \mathrm{m})$ between cementoenamel junction and alveolar bone crest (CEJ-ABC) as described previously (28). In addition, bone volume fraction, represented as bone volume/tissue volume, of the region of interest (ROI) in the first molar beneath the furcation area were evaluated using the CT-Analyser 1.13 software (SCANCO Medical AG). The alveolar bone loss was evaluated by a single examiner who was blinded to the experimental design.

Leukocyte acid phosphatase (TRAP) staining. For the quantification of osteoclasts, a TRAP kit (Sigma Aldrich;
Table I. Changes in the BW of rats during the 6-week experimental period.

\begin{tabular}{lccc}
\hline Study group & $\begin{array}{c}\text { Initial } \\
\text { BW, }\end{array}$ & $\begin{array}{c}\text { Terminal } \\
\text { BW, g }\end{array}$ & $\begin{array}{c}\text { Average BW } \\
\text { gain/day, g }\end{array}$ \\
\hline Control & $291 \pm 5$ & $371 \pm 2.6$ & $2.9 \pm 0.1$ \\
Ligation & $293 \pm 4$ & $359 \pm 2.6$ & $2.4 \pm 0.2$ \\
Ligation + BA12.5 & $290 \pm 7$ & $366 \pm 6.1$ & $2.7 \pm 0.1$ \\
Ligation + BA25 & $286 \pm 4$ & $365 \pm 2.3$ & $2.8 \pm 0.2$ \\
Ligation + BA50 & $289 \pm 9$ & $364 \pm 6.4$ & $2.7 \pm 0.5$ \\
\hline
\end{tabular}

Data are presented as the mean $\pm \mathrm{SD}(\mathrm{n}=5)$. BW, body weight; $\mathrm{BA} 12.5$, $12.5 \mathrm{mg} / \mathrm{kg} /$ day biochanin A; BA25, $25 \mathrm{mg} / \mathrm{kg} /$ day biochanin A; BA $50,50 \mathrm{mg} / \mathrm{kg} /$ day biochanin A.

Merck $\mathrm{KGaA}$ ) was used to stain sections according to the manufacturer's protocol. The results were assessed by a light microscope with a camera connected to a computer (IX71; Olympus Corporation). TRAP-positive multinucleated $(\geq 2)$ cells at the alveolar bone surface were defined as osteoclasts (29). The present study used Image-Pro Plus 6.0 software (Media Cybernetics, Inc.) to analyze three fields of each section. Results are reported as the number of positively stained cells per unit area of alveolar bone (number $/ \mathrm{mm}^{2}$ ).

Immunohistochemical analysis of $O C N$ and Nrf2. After the rats' maxilla were decalcified in $10 \%$ EDTA solution for 1 month, the samples were dehydrated, embedded into paraffin blocks and sliced into $4-\mu m$-thick sections for immunohistochemical analysis. The slides were washed twice at $5 \mathrm{~min}$ each in TBS plus $0.025 \%$ Triton X-100 and blocked in 10\% normal goat serum (cat. no. 31873; Invitrogen; Thermo Fisher Scientific, Inc.) with 1\% BSA (cat. no. A2153; Sigma Aldrich; Merck KGaA) solution in TBS for $2 \mathrm{~h}$ at room temperature. Primary antibodies of OCN (1:200; cat. no. ab13420; Abcam) and Nrf2 (1:300; cat. no. ab137550; Abcam) were diluted in TBS plus $1 \%$ BSA. The slides were incubated with the primary antibodies overnight at $4^{\circ} \mathrm{C}$. After $2 \mathrm{X}$ TBS wash at 5 min each, slides were incubated in horseradish peroxidase-linked antibodies (cat. nos. 7076 and 7074, Cell Signaling Technology, Inc.) at room temperature for $15 \mathrm{~min}$. The sections were examined for immunohistochemical analyses three times by the same examiner using the aforementioned IX71 microscope with a camera connected to a computer. Images of three visual fields from each rat were captured. The total count of immuno-positive cells was performed in the complete area of all photographs using Image-Pro Plus 6.0 software.

Statistical analysis. Data are presented as the mean \pm SD and were analyzed using the SPSS software (version 17.0; SPSS, Inc.). One-way ANOVA and Tukey's test was used for statistical analysis and $\mathrm{P}<0.05$ was considered to indicate a statistically significant difference.

\section{Results}

Effects of BA on gingival fibroblast viability. The MTT assay results indicated that $\mathrm{BA}$ at 50 to $150 \mu \mathrm{M}$ induced no significant 

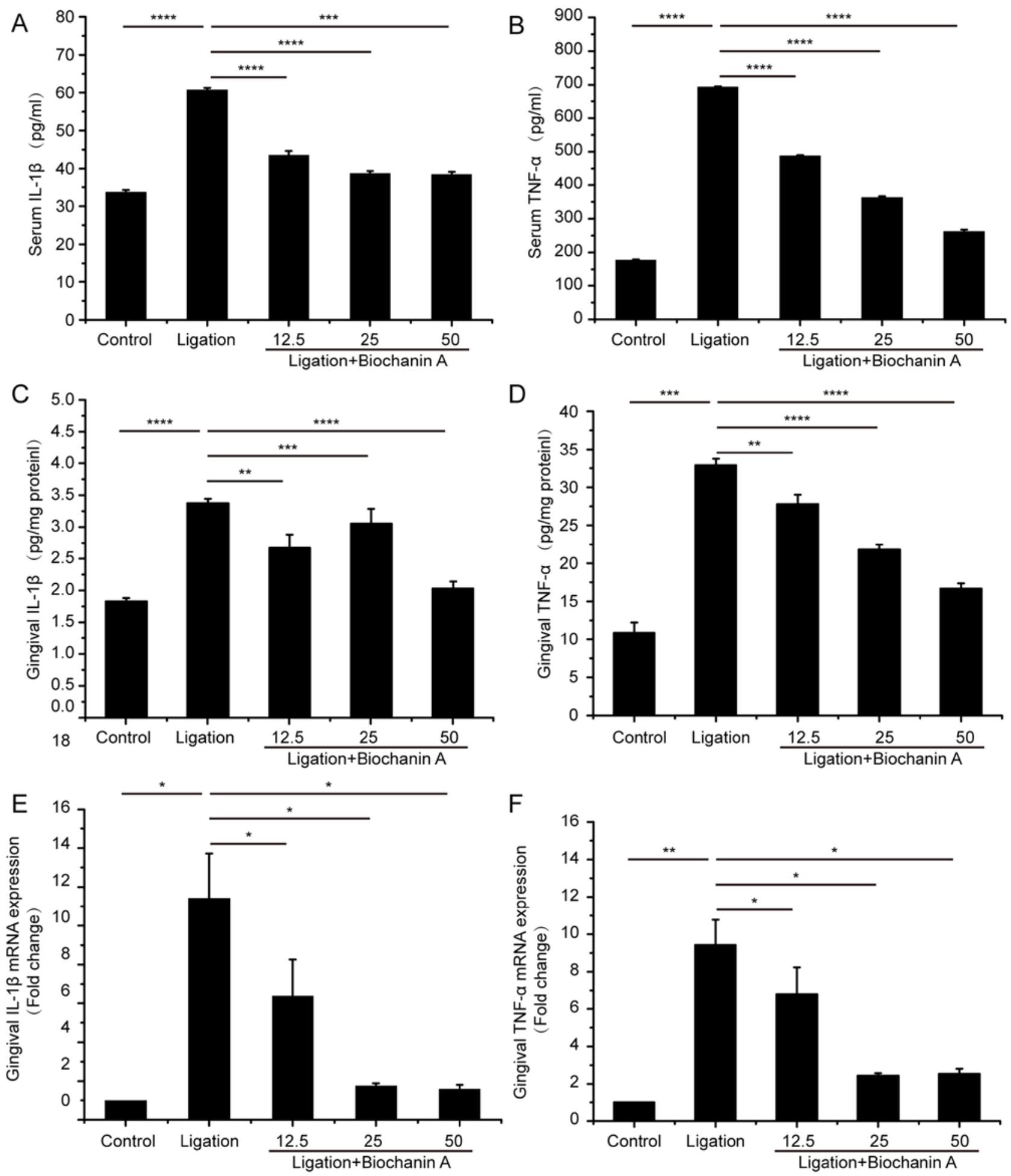

Figure 1. Effects of biochanin A on gingival and serum TNF- $\alpha$ and IL-1 $\beta$ levels. Quantitative analysis of levels of proinflammatory cytokines (A) IL-1 $\beta$ and (B) TNF- $\alpha$ in serum. The levels of (C) IL-1 $\beta$ and (D) TNF-a in gingiva were determined by ELISA. The expression level of (E) IL-1 $\beta$ and (F) TNF- $\alpha$ in gingiva determined using reverse transcription-quantitative PCR. Data are presented as the mean $\pm \mathrm{SD}$. $\mathrm{n}=5$ rats per group. ${ }^{*} \mathrm{P}<0.05,{ }^{* * *} \mathrm{P}<0.01,{ }^{* * * *} \mathrm{P}<0.001$ and ${ }_{* * * * * *} \mathrm{P}<0.0001 .12 .5,12.5 \mathrm{mg} / \mathrm{kg} /$ day biochanin $\mathrm{A} ; 25,25 \mathrm{mg} / \mathrm{kg} / \mathrm{day}$ biochanin $\mathrm{A} ; 50,50 \mathrm{mg} / \mathrm{kg} /$ day biochanin $\mathrm{A}$.

cytotoxicity toward gingival fibroblasts (Fig. S1). Cytotoxicity was represented as the concentration of BA inhibiting cell viability by $50 \%$.

Effects of BA on rat body weight. Body weight monitoring on a weekly basis for six weeks revealed that the body weight of rats was increased by $2.9 \pm 0.1$ and $2.4 \pm 0.2 \mathrm{~g}$ in the control and ligation group, respectively. BA treatment groups showed a higher gain in the body weight compared with the ligation group (Table I); however, the differences were not statistically significant.

Gingival inflammation and oxidative stress levels are attenuated by $B A$. Serum and gingival IL- $1 \beta$ and TNF- $\alpha$ levels 

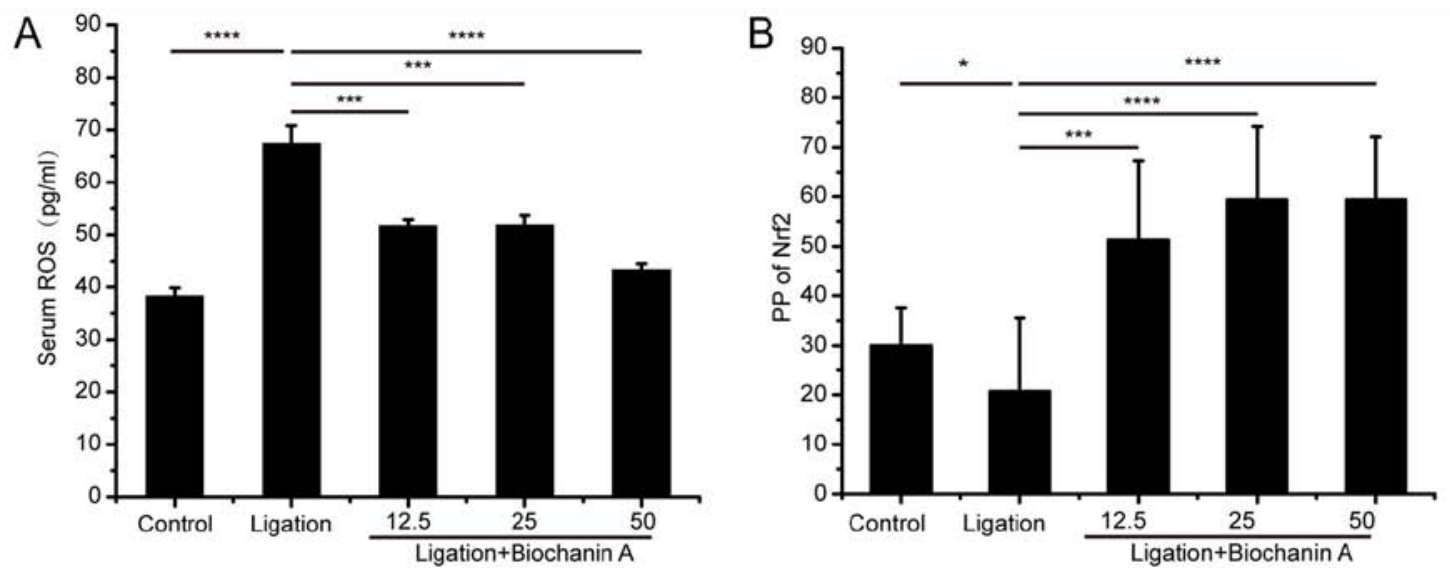

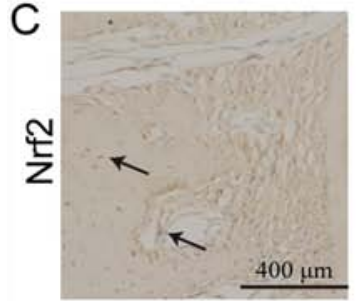

Control

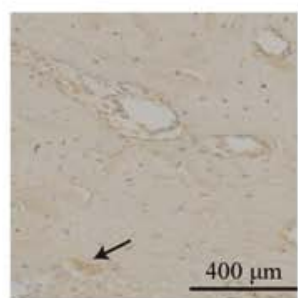

Ligation

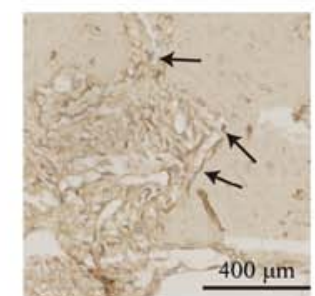

Ligation+BA12.5
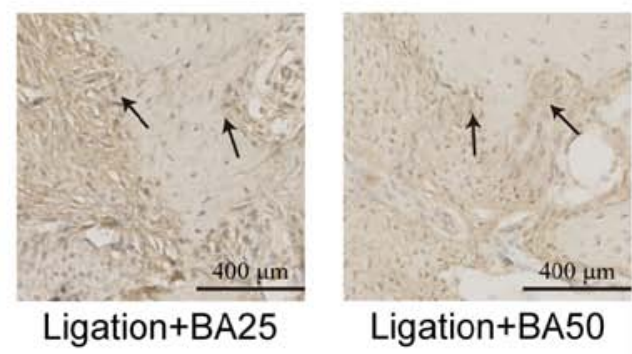

Ligation+BA50

Figure 2. Effect of biochanin A on oxidative stress levels. (A) Effect of biochanin A on serum ROS levels. (B and C) Effects of BA on Nrf2 expression in the alveolar bone of rats determined by immunohistochemical staining. Nrf2-positive cells are indicated with black arrows. Data are presented are the mean \pm SD. $\mathrm{n}=5$ rats per group. ${ }^{*} \mathrm{P}<0.05,{ }^{* * *} \mathrm{P}<0.001$ and ${ }^{* * * * *} \mathrm{P}<0.0001 . \mathrm{BA} 12.5,12.5 \mathrm{mg} / \mathrm{kg} /$ day biochanin A; BA25, $25 \mathrm{mg} / \mathrm{kg} / \mathrm{day}$ biochanin A; BA50, $50 \mathrm{mg} / \mathrm{kg} / \mathrm{day}$ biochanin A; PP, percentage of positive cells.

were significantly increased in the ligation group compared with the control group (Fig. 1A-D). Treatment with BA significantly inhibited the serum TNF- $\alpha$ and IL- $1 \beta$ levels induced by experimental periodontitis. Moreover, gingival mRNA levels of TNF- $\alpha$ and IL-1 $\beta$ were also alleviated with BA treatment (Fig. 1E and F). In addition, serum ROS level was significantly higher in the ligation group compared with the control group. However, treatment with BA inhibited ligation-induced ROS levels (Fig. 2A). The analysis of effects of BA on Nrf2 expression by immunohistochemistry showed that the expression of $\mathrm{Nfr} 2$ in gingival tissues was increased following BA treatment (Fig. 2B and C).

BA alleviates bone loss. Histological analysis of control group specimens showed normal physiological periodontium including clearly defined gingiva, periodontal ligament, cementum and alveolar bone (Fig. 3A). By contrast, the ligation group showed absorption in the alveolar bone between first and second molars. The gingival epithelial spikes hyperplasia, thickened stratum spinosum and marked breakdown of collagen fibers could also be observed (Fig. 3A). The treatment with BA apparently recovered tissue degradation in the ligation group (Fig. 3A). The micro-CT analyses indicated that the medium and high dose of BA-treated groups showed shorter distances of CEJ-ABC compared with those in the ligation group (Fig. $3 \mathrm{~B}$ and $\mathrm{C}$ ). The bone volume fraction beneath the furcation area of maxillary first molar increased in all three BA-treated groups compared with that in the ligation group (Fig. 3D); however the levels observed in the control group have not been fully restored (Fig. 3B-D).
BA affects bone turnover and metabolism. The ligation group showed a greater number of TRAP-positive osteoclasts compared with the control group (Fig. 4A). There was also marked difference in the OCN level between the control and the ligation group (Fig. 4B). Medium and high doses of BA treatment significantly decreased the TRAP-positive osteoclast numbers in the alveolar bone of rats with experimental periodontitis (Fig. 4C). Moreover, treatment with a high dose of BA increased the serum OCN level compared with the ligation group (Fig. 4D).

\section{Discussion}

The present study investigated the role of BA administration for the management of gingival inflammation and alveolar bone destruction associated with periodontitis. For this purpose, effects of BA supplementation on oxidative stress levels, various biomarkers associated with periodontitis, including IL-1 $\beta$, TNF- $\alpha$ and OCN, and bone loss were analyzed.

Ligature-induced periodontitis model is commonly used and can induce soft tissue trauma, masticatory discomfort or periodontal infection. A stress response, resulting in postoperative weight loss, may also be observed in rat with experimental periodontitis induced by LPS (30). A previous study has shown that under stress, obese animals exhibit a more severe weight loss compared with normal weight control groups, and it is difficult to recover weight loss after repeated stress (31). However, in the present study, ligation placed around the first molars did not significantly influence the weight gain compared to that in the control group. A possible explanation is that compared with injection of LPS, ligation 

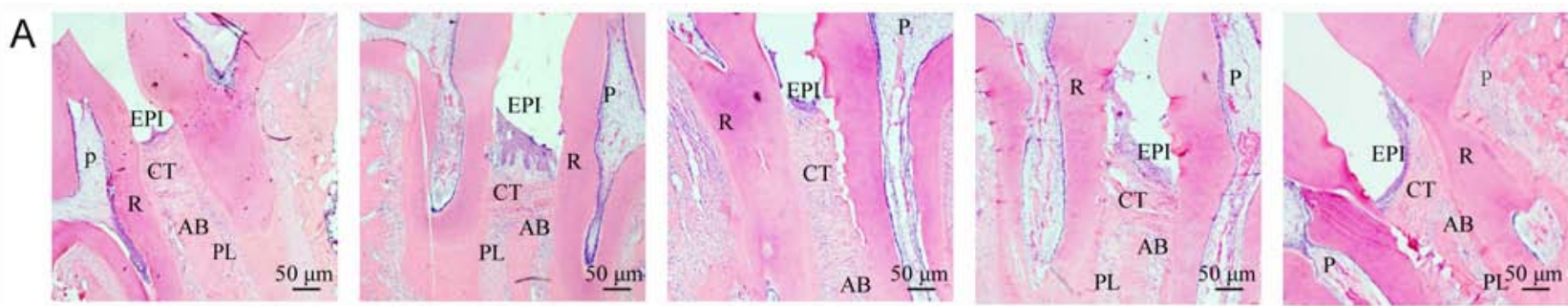

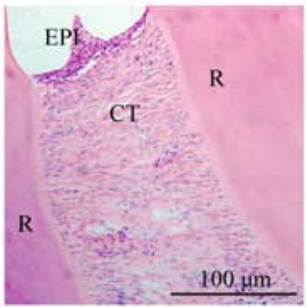

Control

B
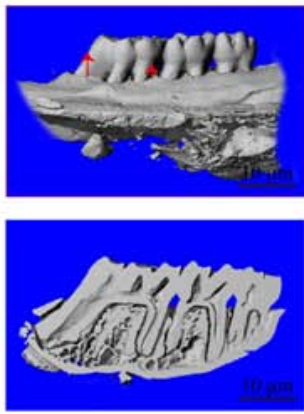

Control

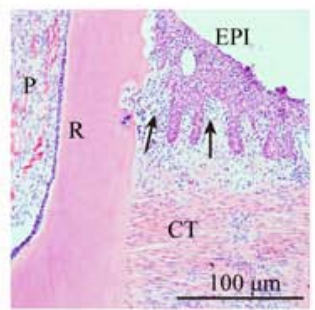

Ligation
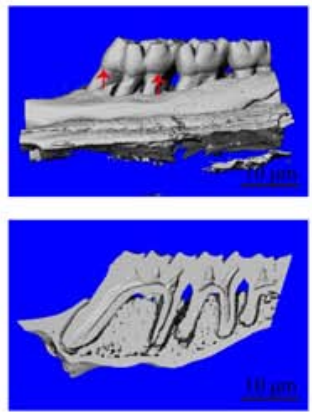

Ligation

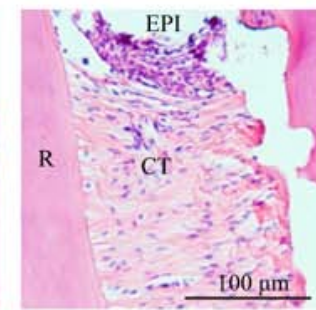

Ligation+BA12.5
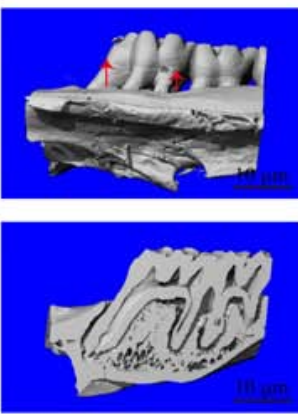

Ligation+BA12.5

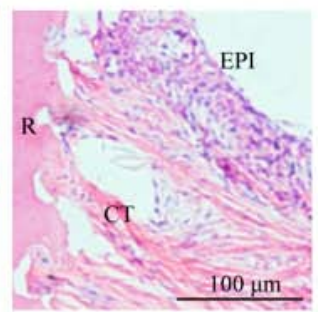

Ligation+BA25
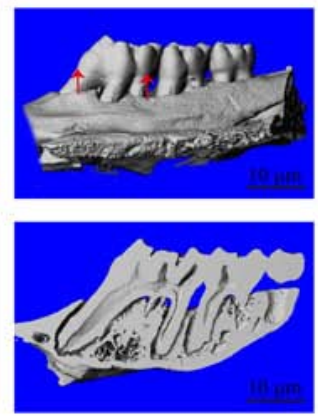

Ligation+BA25

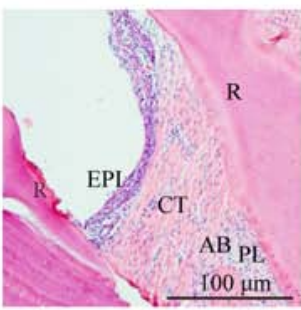

Ligation+BA50
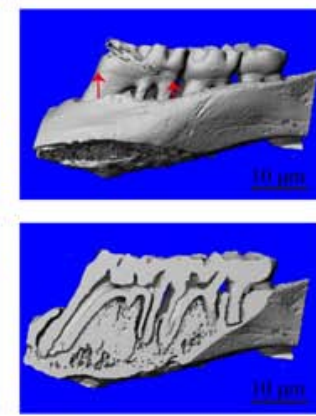

Ligation+BA50
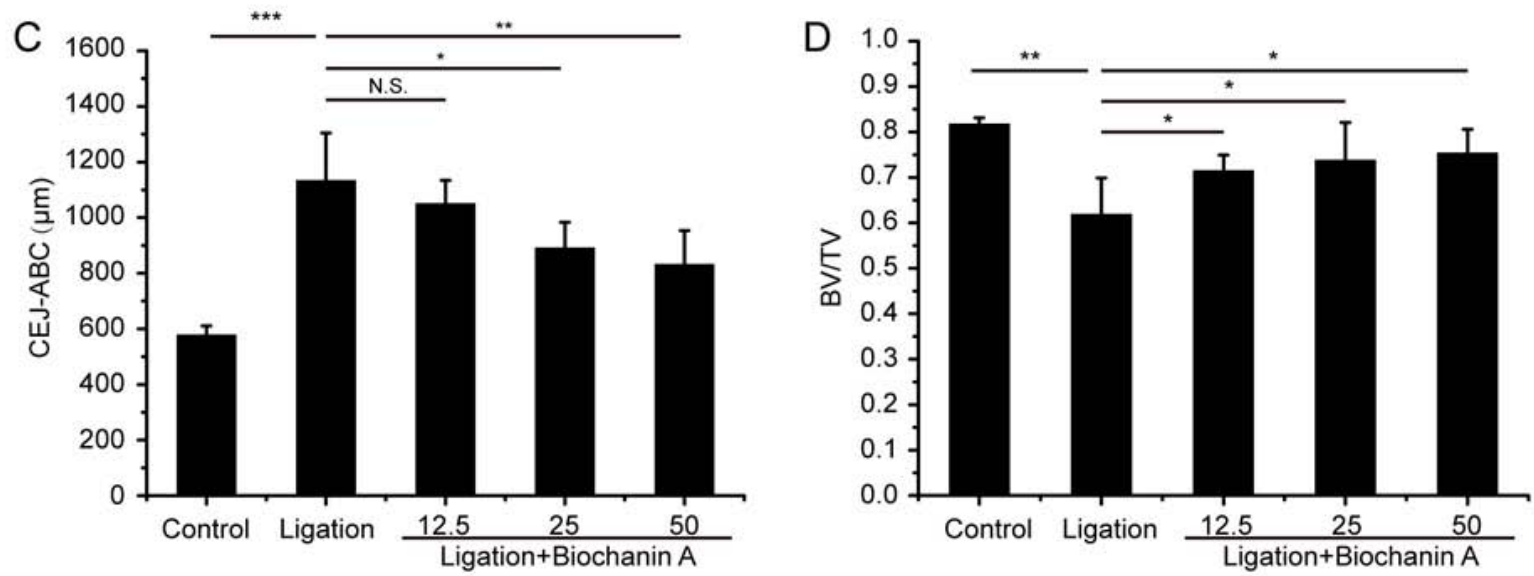

Figure 3. Role of biochanin A in ameliorating the alveolar bone loss in rats with periodontitis. (A) Hematoxylin and eosin staining indicated periodontal morphology. Images on the bottom row magnifies 2.5 times of the regions of interest on the top row. PL, periodontal ligament; R, root; AB, alveolar bone; EPI, oral epithelium; CT, connective tissue; P, pulp. The black arrow denotes the gingival epithelial spikes hyperplasia. (B) Micro-computed tomography reconstruction shows alveolar bone loss of maxillary first molars around mesial/distal aspect (buccal view). (C) Quantitative analysis of bone loss around mesial/distal aspect. (D) Quantitative analysis of BV of the region of interest in the first molar beneath the furcation area. Data are presented as the mean \pm SD, $\mathrm{n}=5$ rats per group. ${ }^{*} \mathrm{P}<0.05,{ }^{* *} \mathrm{P}<0.01,{ }^{* * *} \mathrm{P}<0.001$ and $\mathrm{N}$.S. means no significant difference. CEJ-ABC, cemento-enamel junction and alveolar bone crest; BA12.5, $12.5 \mathrm{mg} / \mathrm{kg} /$ day biochanin A; BA25, $25 \mathrm{mg} / \mathrm{kg} /$ day biochanin A; BA50, $50 \mathrm{mg} / \mathrm{kg} /$ day biochanin A; BV/TV, bone volume/tissue volume.

alone for 6 weeks can only gradually cause local periodontal destruction by mechanical injurywithout affecting food intake or body weight, consistent with a previous study (32).

Inflammatory response and oxidative stresses play an important role in the pathogenesis of periodontitis $(16,33)$; therefore, host immune regulatory drugs may be considered as an auxiliary treatment option for periodontitis management. The current study used BA, an isoflavone compound which acts as a competitive substrate for various enzymes and has been demonstrated to bind to proteins and DNA $(34,35)$. In addition, a number of studies showed that BA played an anti-inflammatory and antioxidative role in various conditions including cancer, heart disease, perimenopausal syndrome and osteoporosis (2-4). These findings suggested that BA can be a potential anti-inflammatory and anti-oxidative agent for protecting the periodontal tissues against inflammatory destruction. However, these results require further investigation. Biomarkers such as IL-1 $\beta$ and TNF- $\alpha$ are 


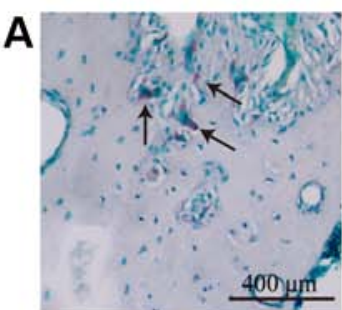

Control

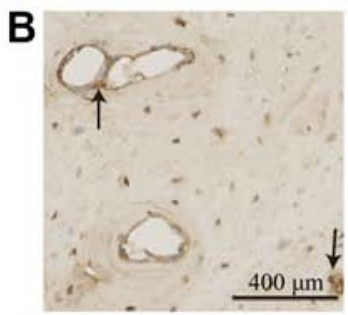
Control

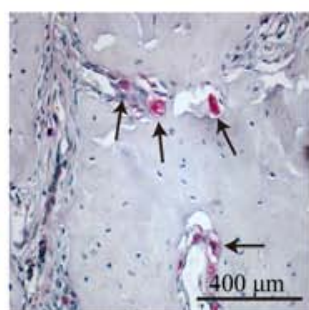

Ligation

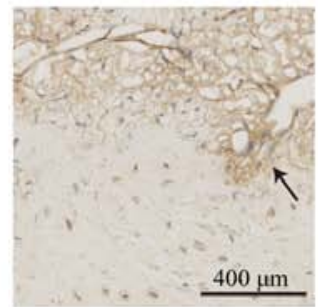

Ligation
C

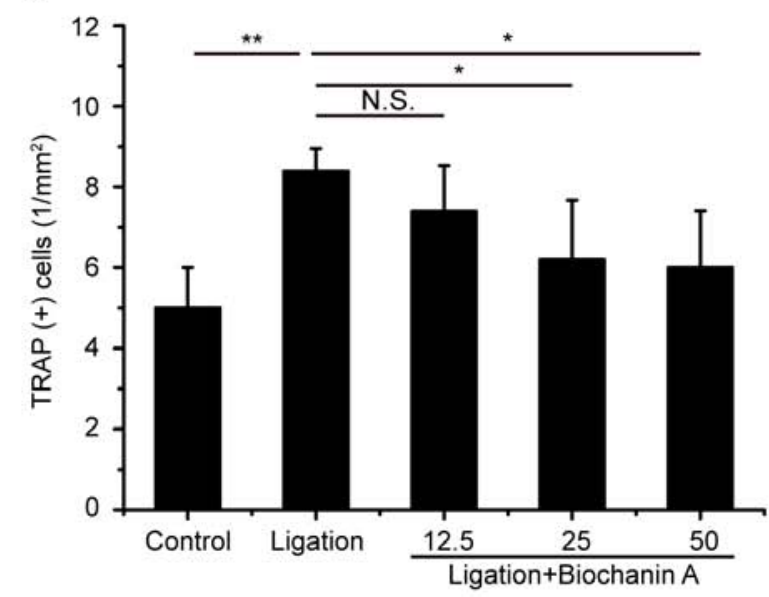

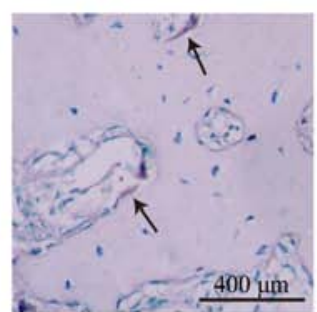

Ligation+BA12.5

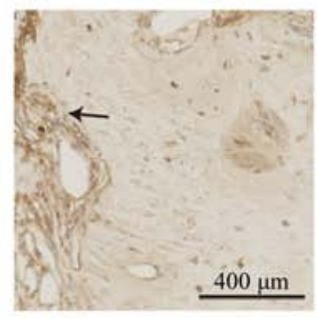

Ligation+BA12.5

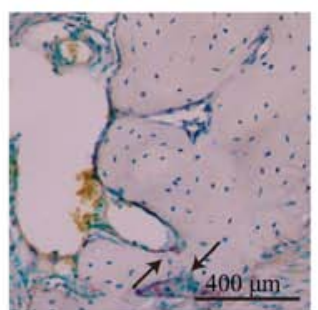

Ligation+BA25

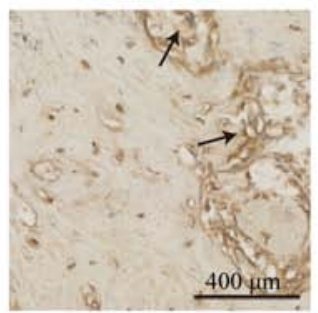

Ligation+BA25

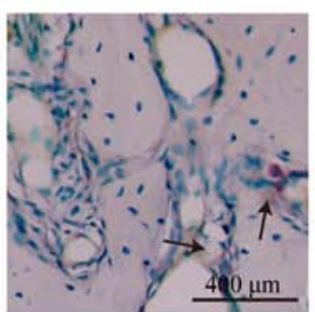

Ligation+BA50

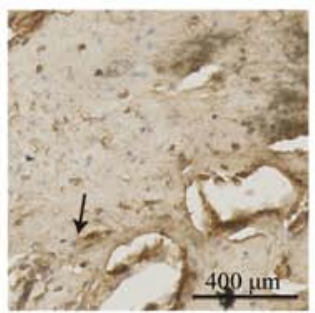

Ligation+BA50

D

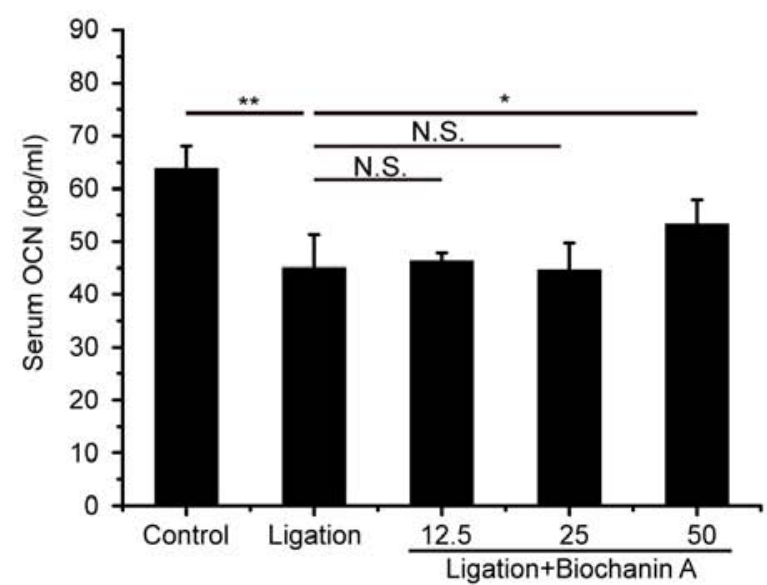

Figure 4. Effects of biochanin A on bone turnover and metabolism. (A) Effects of biochanin A on osteoclast TRAP staining. TRAP ${ }^{+}$cells are indicated with black arrows. (B) Effects of biochanin A on OCN in the alveolar bone of rats determined by immunohistochemical staining. The black arrows indicate the IHC positive cells. (C) Quantitative analysis of TRAP ${ }^{+}$cells in five groups. (D) Effects of BA on OCN in serum of rats determined by ELISA. Data are presented as the mean $\pm \mathrm{SD}, \mathrm{n}=5$ rats per group. ${ }^{*} \mathrm{P}<0.05,{ }^{* *} \mathrm{P}<0.01$, and N.S. means no significant difference. TRAP, leukocyte acid phosphatase; $\mathrm{BA} 12.5,12.5 \mathrm{mg} / \mathrm{kg} / \mathrm{day}$ biochanin A; BA25, $25 \mathrm{mg} / \mathrm{kg} /$ day biochanin A; BA50, $50 \mathrm{mg} / \mathrm{kg} /$ day biochanin A.

considered osteoresorptive factors promoting bone loss, osteoclastogenesis and bone resorption through stimulation of osteoclast maturation and receptor activator of nuclear factor- $\kappa \mathrm{B}$ (RANK) ligand (RANKL) in osteoblasts (36). The present study revealed that TNF- $\alpha$ and IL-1 $\beta$ levels were reduced in serum and gingival tissues of rats in groups treated with BA. Therefore, the inhibitory role of BA on bone resorption may be associated with its anti-inflammatory effects. The tissue destruction in periodontitis is mainly caused by an imbalance between periodontal pathogens and the host defense, which leads to an aberrant inflammatory response and release of enzymes by neutrophils and ROS for a prolonged period of time (37). In the present study, oxidative stress was measured via ROS levels in serum. In the ligation group, the mean ROS level was increased, confirming that periodontitis was accompanied by ROS activity. However, compared with the ligation group, a significantly lower level of serum ROS was observed following BA supplementation. These findings suggested that treatment with BA is likely to attenuate the overproduction of ROS.
The redox-sensitive transcription factor Nrf2 plays an important role in the antioxidant signaling pathway through inducing the expression of a number of cytoprotective proteins, including heme-oxygenasae-1, glutamate-cysteine ligase catalytic subunit, quinone oxidoreductase-1 and nicotinamide adenine dinucleotide phosphate via an antioxidant response element (ARE) (38). Since BA exhibits antioxidative characteristics to inhibit oxidative stresses in the pathogenesis of periodontitis, the present study examined the Nrf2-positive cell counts in the alveolar bone of rats determined by immunohistochemical staining to further explore the underlying mechanisms at the molecular level. The current study revealed that BA treatment significantly improved the number of Nrf2-positive cells in the alveolar bone. These results are consistent with a recent study by Liang et al (39), where BA was an effective Nfr2 activator. In addition, BA has the capability to counteract oxidative damages induced by LPS $(33,40)$, high-fat diet (12) and D-galactosamine (41). A previous in vitro study reported that BA promoted the nuclear accumulation of $\mathrm{Nrf} 2$ and enhanced 
its binding activity with ARE, hence upregulating the expression of antioxidative and cytoprotective enzymes (42). To the best of our knowledge, the current study is the first to report that BA protected periodontal tissues from oxidative damage via enhancing the expression of $\mathrm{Nfr} 2$ in rats with experimental periodontitis.

Alveolar bone resorption is another characteristic feature of periodontitis (43). The present study used micro-CT to evaluate the effectiveness of BA in preventing bone loss in rats with periodontitis. It was observed that the alveolar bone volume beneath the furcation area was increased in all rats treated with BA compared with the ligation group. The protective effect of high dose of BA was manifested by an increased level of OCN. Furthermore, it was found that medium and high dose BA markedly attenuated the number of TRAP-positive cells, which indicate that BA may suppress osteoclastic growth and activity resulting in a reduced bone turnover. BA administered at a dose of $50 \mathrm{mg} / \mathrm{kg}$ per/day exerted the greatest therapeutic effect. These results are consistent with a previous study were BA had positive effects on bone loss in ovariectomized rats (4). Alveolar bone resorption is driven by osteoclasts (44). As a key osteoclastogenic cytokine receptor activator, RANKL can be recognized by receptor activator of RANK, thus inducing the differentiation of bone marrow macrophages into osteoclasts (45). IL-1 $\beta$ and TNF- $\alpha$ have been implicated in stimulating osteoblasts to express RANKL, which in turn induces the differentiation of osteoclasts $(44,46)$.

Furthermore, ROS acts as an intracellular signaling molecule, which can active NF- $\mathrm{BB}$ and cause bone destruction via osteoclastogenesis (47). BA had a significant effect on the production of IL- $1 \beta$, TNF- $\alpha$ and ROS in the current study. Considering the association between proinflammatory cytokines and RANKL as well as ROS and RANKL, BA may attenuate alveolar bone resorption by reducing oxidative stress. The possible effect of BA on markers of alveolar bone turnover and metabolism, including RANKL/osteoprotegerin, osterix, transforming growth factor $\beta$ and ALP, should be further studied.

In conclusion, the present study used an animal model to investigate the effects of BA against experimental periodontitis induced by ligation. BA can inhibit inflammation and regulate unbalanced oxidative stress response and ameliorate the alveolar bone loss. Therefore, BA represents a promising adjunctive therapy and can be used to modulate host response to periodontitis. Although the present study provided an insight into the potential applications of BA for the treatment of periodontitis, further molecular studies and clinical trials are required before it can be used in clinical practice.

\section{Acknowledgements}

Not applicable.

\section{Funding}

This work was supported by the National Natural Science Foundation of China (grant no. 81600871) and Fundamental Research Funds for the Central Universities (2019SCU12062).

\section{Availability of data and materials}

The datasets used and/or analyzed during the current study are available from the corresponding author on reasonable request.

\section{Authors' contributions}

SZ performed the majority of the experiments and produced the figures. YN analyzed majority of the data and wrote the first draft of the manuscript. ZY constructed the animal models. YZ completed the ELISA experiments. QG collected and analyzed micro-CT data. YY and XZ contributed to the conception and design of the work and revised the manuscript critically for important intellectual content and contributed to the discussion. YD and CL contributed to conception, design, data acquisition, analysis, and interpretation, drafted and critically revised the manuscript for important intellectual content. All authors read and approved the final version of the manuscript.

\section{Ethics approval and consent to participate}

The present study was approved by Ethics Committee of the State Key Laboratory of Oral Diseases, West China Hospital of Stomatology, Sichuan University (approval no. WCHSIRB-D-2018-148).

\section{Patient consent for publication}

Not applicable.

\section{Competing interests}

The authors declare that they have no competing interests.

\section{References}

1. Renda G, Yalçın FN, Nemutlu E, Akkol EK, Süntar İ, Keleş H, Ina H, Çalış İ and Ersöz T: Comparative assessment of dermal wound healing potentials of various Trifolium L. Extracts and determination of their isoflavone contents as potential active ingredients. J Ethnopharmacol 148: 423-432, 2013.

2. Srinivas NR: Biochanin A: Understanding the complexities in the paradoxical drug-drug interaction potential. Eur J Drug Metab Pharmacokinet 40: 119-125, 2015.

3. Puthli A, Tiwari R and Mishra KP: Biochanin a enhances the radiotoxicity in colon tumor cells in vitro. J Environ Pathol Toxicol Oncol 32: 189-203, 2013.

4. Su SJ, Yeh YT and Shyu HW: The preventive effect of biochanin a on bone loss in ovariectomized rats: Involvement in regulation of growth and activity of osteoblasts and osteoclasts. Evid Based Complement Alternat Med 2013: 594857, 2013.

5. Chen HQ, Jin ZY and Li GH: Biochanin A protects dopaminergic neurons against lipopolysaccharide-induced damage through inhibition of microglia activation and proinflammatory factors generation. Neurosci Lett 417: 112-117, 2007.

6. Kole L, Giri B, Manna SK, Pal B and Ghosh S: Biochanin-A, an isoflavon, showed anti-proliferative and anti-inflammatory activities through the inhibition of iNOS expression, p38-MAPK and ATF-2 phosphorylation and blocking NFKB nuclear translocation. Eur J Pharmacol 653: 8-15, 2011.

7. Ming X, Ding M, Zhai B, Xiao L, Piao T and Liu M: Biochanin A inhibits lipopolysaccharide-induced inflammation in human umbilical vein endothelial cells. Life Sci 136: 36-41, 2015.

8. Lee KH and Choi EM: Biochanin A stimulates osteoblastic differentiation and inhibits hydrogen peroxide-induced production of inflammatory mediators in MC3T3-E1 cells. Biol Pharm Bull 28: 1948-1953, 2005. 
9. Oh JS, Cho IA, Kang KR, You JS, Yu SJ, Lee GJ, Seo YS, Kim CS, Kim DK, Kim G, et al: Biochanin-A antagonizes the interleukin-1 $\beta$-induced catabolic inflammation through the modulation of $\mathrm{NF \kappa B}$ cellular signaling in primary rat chondrocytes. Biochem Biophys Res Commun 477: 723-730, 2016.

10. Vanden Berghe W, Dijsselbloem N, Vermeulen L, Ndlovu MN Boone $\mathrm{E}$ and Haegeman G: Attenuation of mitogen- and stress-activated protein kinase-1-driven nuclear factor-kappaB gene expression by soy isoflavones does not require estrogenic activity. Cancer Res 66: 4852-4862, 2006.

11. Wu LY, Ye ZN, Zhuang Z, Gao Y, Tang C, Zhou CH, Wang CX, Zhang XS, Xie GB, Liu JP, et al: Biochanin A Reduces inflammatory injury and neuronal apoptosis following subarachnoid hemorrhage via suppression of the TLRs/TIRAP/MyD88/NF- $\mathrm{B}$ pathway. Behavioural Neurology 2018: 1-10, 2018.

12. Xue Z, Zhang Q, Yu W, Wen H, Hou X, Li D and Kou X Potential lipid-lowering mechanisms of biochanin A. J Agric Food Chem 65: 3842-3850, 2017.

13. Ko WC, Lin LH, Shen HY, Lai CY, Chen CM and Shih $\mathrm{CH}$ Biochanin a, a phytoestrogenic isoflavone with selective inhibition of phosphodiesterase 4, suppresses ovalbumin-induced airway hyperresponsiveness. Evid Based Complement Alternat Med 2011: 635058, 2011.

14. Beck V, Rohr U and Jungbauer A: Phytoestrogens derived from red clover: An alternative to estrogen replacement therapy? J Steroid Biochem Mol Biol 94: 499-518, 2005.

15. GBD 2016 Disease and Injury Incidence and Prevalence Collaborators: Global, regional, and national incidence, prevalence, and years lived with disability for 328 diseases and injuries for 195 countries, 1990-2016: A systematic analysis for the Global Burden of Disease Study 2016. Lancet 390: 1211-1259, 2017.

16. Darveau RP: Periodontitis: A polymicrobial disruption of host homeostasis. Nat Rev Microbiol 8: 481-490, 2010.

17. Liu C, Mo L, Niu Y, Li X, Zhou X and Xu X: The role of reactive oxygen species and autophagy in periodontitis and their potential linkage. Front Physiol 8: 439, 2017

18. Hanazawa S, Nakada K, Ohmori Y, Miyoshi T, Amano S and Kitano S: Functional role of interleukin 1 in periodontal disease: Induction of interleukin 1 production by Bacteroides gingivalis lipopolysaccharide in peritoneal macrophages from $\mathrm{C} 3 \mathrm{H} / \mathrm{HeN}$ and C3H/HeJ mice. Infect Immun 50: 262-270, 1985.

19. Nibali L and Donos N: Periodontitis and redox status: A review. Curr Pharm Des 19: 2687-2697, 2013.

20. Chapple IL and Matthews JB: The role of reactive oxygen and antioxidant species in periodontal tissue destruction. Periodontol 43: 160-232, 2010.

21. Dursun E, Akalin FA, Genc T, Cinar N, Erel O and Yildiz BO Oxidative stress and periodontal disease in obesity. Medicine (Baltimore) 95: e3136, 2016.

22. Soares ASLS, Scelza MZ, Spoladore J, Gallito MA, Oliveira F, Moraes RCM and Alves GG: Comparison of primary human gingival fibroblasts from an older and a young donor on the evaluation of cytotoxicity of denture adhesives. J Appl Oral Sci 26: e20160594, 2018.

23. Chen H, Shi Q, Qing Y, Yao YC and Cao YG: Cytotoxicity of modified nonequilibrium plasma with chlorhexidine digluconate on primary cultured human gingival fibroblasts. J Huazhong Univ Sci Technolog Med Sci 36: 137-141, 2016.

24. Wu DQ, Zhong HM, Ding QH and Ba L: Protective effects of biochanin A on articular cartilage: In vitro and in vivo studies. BMC Complement Altern Med 14: 444, 2014.

25. Abe T and Hajishengallis G: Optimization of the ligature-induced periodontitis model in mice. J Immunol Methods 394: 49-54, 2013.

26. Livak KJ and Schmittgen TD: Analysis of relative gene expression data using real-time quantitative PCR and the 2(-Delta Delta C(T)) method Methods 25: 402-408, 2001

27. Bhattarai G, Kook SH, Kim JH, Poudel SB, Lim SS, Seo YK and Lee JC: COMP-Ang1 prevents periodontitic damages and enhances mandible bone growth in an experimental animal model. Bone 92: 168-179, 2016.

28. Papathanasiou E, Kantarci A, Konstantinidis A, Gao H and Van Dyke TE: SOCS-3 regulates alveolar bone loss in experimental periodontitis. J Dent Res 95: 1018-1025, 2016.
29. Li CH and Amar S: Morphometric, histomorphometric, and microcomputed tomographic analysis of periodontal inflammatory lesions in a murine model. J Periodontol 78: 1120-1128, 2007.

30. Dumitrescu AL, Abd-El-Aleem S, Morales-Aza B and Donaldson LF: A model of periodontitis in the rat: Effect of lipopolysaccharide on bone resorption, osteoclast activity, and local peptidergic innervation. J Clin Periodontol 31: 596-603, 2004.

31. Lawrence CB, Brough D and Knight EM: Obese mice exhibit an altered behavioural and inflammatory response to lipopolysaccharide. Dis Model Mech 5: 649-659, 2012.

32. Rettori E, De Laurentiis A, Zorrilla Zubilete M, Rettori V and Elverdin JC: Anti-inflammatory effect of the endocannabinoid anandamide in experimental periodontitis and stress in the rat. Neuroimmunomodulation 19: 293-303, 2012.

33. Wang J, He C, Wu WY, Chen F, Wu YY, Li WZ, Chen HQ and Yin YY: Biochanin A protects dopaminergic neurons against lipopolysaccharide-induced damage and oxidative stress in a rat model of Parkinson's disease. Pharmacol Biochem Behav 138 96-103, 2015

34. Luo Q, Shi X, Ding J, Ma Z, Chen X, Leng Y, Zhang X and Liu Y: Network pharmacology integrated molecular docking reveals the antiosteosarcoma mechanism of biochanin A. Evid Based Complement Alternat Med 2019: 1410495, 2019.

35. Nestel P, Cehun M, Chronopoulos A, DaSilva L, Teede H and McGrath B: A biochanin-enriched isoflavone from red clover lowers LDL cholesterol in men. Eur J Clin Nutr 58: 403-408, 2004.

36. Tanaka Y, Nakayamada S and Okada Y: Osteoblasts and osteoclasts in bone remodeling and inflammation. Curr Drug Targets Inflamm Allergy 4: 325-328, 2005.

37. Brock GR, Butterworth CJ, Matthews JB and Chapple IL: Local and systemic total antioxidant capacity in periodontitis and health. J Clin Periodontol 31: 515-521, 2010.

38. Liu Q, Hu Y, Cao Y, Song G, Liu Z and Liu X: Chicoric acid ameliorates lipopolysaccharide-induced oxidative stress via promoting the Keap1/Nrf2 transcriptional signaling pathway in BV-2 microglial cells and mouse brain. J Agric Food Chem 65: 338-347, 2017.

39. Liang F, Cao W, Huang Y, Fang Y, Cheng Y, Pan S and Xu X: Isoflavone biochanin A, a novel nuclear factor erythroid 2-related factor 2 (Nrf2)-antioxidant response element activator, protects against oxidative damage in HepG2 cells. Biofactors 45: 563-574, 2019.

40. Wang J, Wu WY, Huang H, Li WZ, Chen HQ and Yin YY: Biochanin a protects against lipopolysaccharide-induced damage of dopaminergic neurons both in vivo and in vitro via inhibition of microglial activation. Neurotox Res 30: 486-498, 2016.

41. Liu X, Wang T, Liu X, Cai L, Qi J, Zhang P and Li Y: Biochanin A protects lipopolysaccharide/D-galactosamine-induced acute liver injury in mice by activating the Nrf 2 pathway and inhibiting NLRP3 inflammasome activation. Int Immunopharmacol 38: 324-331, 2016.

42. Kim J, Cha YN and Surh YJ: A protective role of nuclear factor-erythroid 2-related factor-2 (Nrf2) in inflammatory disorders. Mutat Res 690: 12-23, 2010

43. Bostanci N, Abe T, Belibasakis GN and Hajishengallis G: TREM-1 Is upregulated in experimental periodontitis, and its blockade inhibits IL-17A and RANKL expression and suppresses bone loss. J Clin Med 8: 1579, 2019.

44. Hienz SA, Sweta P and Saso I: Mechanisms of bone resorption in periodontitis. J Immunol Res 2015: 615486, 2015.

45. Teitelbaum SL: Bone resorption by osteoclasts. Science 289 : 1504-1508, 2000.

46. Feng W, Guo J and Li M: RANKL-independent modulation of osteoclastogenesis. J Oral Biosci 61: 16-21, 2019.

47. Sağlam M, Köseoğlu S, Hatipoğlu M, Esen HH and Köksal E: Effect of sumac extract on serum oxidative status, RANKL/OPG system and alveolar bone loss in experimental periodontitis in rats. J Appl Oral Sci 23: 33-41, 2015.

This work is licensed under a Creative Commons Attribution-NonCommercial-NoDerivatives 4.0 International (CC BY-NC-ND 4.0) License. 